\title{
BIOACTIVITY STUDIES OF SOLANUM FEROX L. AGAINST TRIBOLIUM CASTANEUM (HERBST) ADULTS
}

\author{
M. Abdullah, M. Nesa, R. Islam, J. Banu, J. Sarkar and N. Islam ${ }^{1}$ \\ Department of Zoology, University of Rajshahi, Rajshahi-6205, Bangladesh \\ ${ }^{1}$ Corresponding author, E-mail:n_islamm@yahoo.com; nurulislam@ru.ac.bd
}

\begin{abstract}
Dose-mortality and repellent activity tests of the chloroform extracts of fruit, leaf, root and stem of Solanum ferox L. against the red flour beetle Tribolium castaneum (Herbst) adults have been done. The fruit, leaf and stem extracts offered promising toxicity to the beetles within $30 \mathrm{~min}$. of exposure. The $\mathrm{LD}_{50}$ values for $30 \mathrm{~min} ., 12,24,36$ and $48 \mathrm{~h}$ of exposures were $0.440,0.247,0.211,0.207$ and $0.164 \mathrm{mg} \mathrm{cm}^{-2}$ respectively for the fruit extract; 2.394, 1.712, $1.540,1.239$ and $0.993 \mathrm{mg} \mathrm{cm}^{-2}$ respectively for the leaf extract; and $4.918,0.871,0.546,0.472$ and $0.522 \mathrm{mg} \mathrm{cm}^{-2}$ respectively for the stem extract. However, the root extract did not offer any mortality for $30 \mathrm{~min}$. and $12 \mathrm{~h}$ of exposure; and thus the $\mathrm{LD}_{50}$ values for 24,36 and $48 \mathrm{~h}$ of exposure were $6.667,5.086$ and $3.357 \mathrm{mg} \mathrm{cm}^{-2}$ respectively. While the dose-mortality activity can be arranged in the order of fruit> leaf $>$ stem $>$ root extracts. The fruit and stem extracts showed repellent activity against $T$. castaneum adults at $\mathrm{P}<0.01$ and the leaf extract at $\mathrm{P}<0.05$ level of significance, while the root extract did not show any repellency.
\end{abstract}

Keywords: Mortality, repellency, Solanum ferox, Tribolium castaneum.

সারাংশ: পূর্ণাঙ Tribolium castaneum (Herbst) এর উপর Solanum ferox L. এর ফল, পাতা, মূল ও কান্ডের ক্লোরোফরম নির্যাস প্রয়োগে মাত্রা-মৃত্যুহার ও বিতাড়ন কার্যকারিতা পর্যবেক্ষণ করা হয়। ফল, পাতা ও কান্ডের নির্যাস প্রয়োগের ৩০ মিনিটের মধ্যেই উল্লেখযোগ্য বিষক্রিয়া দেখায়। ফলের নির্যালের জন্য ৩০মি: এবং ১২,

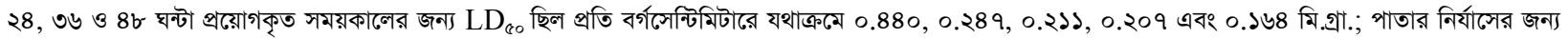

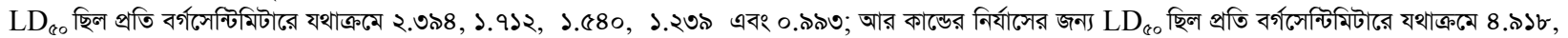
০.৮৭১, ০.৫৪৬, ০.৪৭২ এবং ০.৫২২ মি.গ্রা.। মূলের নির্যাস ১২ ঘন্টা অবধि কোন কার্যকারিতা না দেখালেও ২৪, ৩৬ ও ৪৮- ঘন্টা প্রয়োগকৃত সময়কালের জন্য LD৫० ছিল প্রতি বর্গসেন্টিমিটারে যথাক্রনে ৬.৬৬৭, ৫.০৮৬৬ এবং ৩.৩৫৭ মি.গ্রা.; এবং উপরোক্ত কার্যকারিতার ধারা উচ্চ থেকে নিম্নতর পর্যায়ে ফল> পাতা> কান্ড> মূল এভাবে সাজান

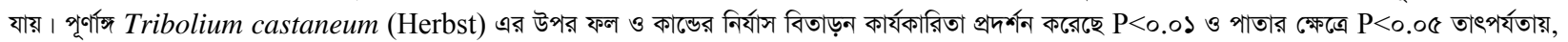
অবশ্য মূলের নির্যাস কোনরূপ কার্যকারিতা দেখায়নি।

\section{Introduction}

Solanum ferox L. of the family Solanaceae is a large valuable annual and perennial plant, small in size, suberect, prickly and hairy herb and gets 0.5 to 1.5 meters in height. They often have attractive fruits and flowers. Most are poisonous but many bear edible fruits, leaves, or tubers, and the genus including three major food crops. A decoction of this root is used against syphilis, for body pains and discomfort after meals, to the cases of different varieties of fevers, as poultice for itches, cuts, wounds and bruises, and as an antipyretic, but for the later it is not supported by the trial conducted to find out the effect of the herb (Kirtikar and Basu, 1935). In Bangladesh, this herb is used for coughs, asthma, fever, vomiting, sore throat and gonorrhea; and in India it is used for female sex disorders. Its roots and berries are bechic, antiasthmatic, antirheumatic, antiviral, anticancer and spermicidal (Joy et al. 2001). However, information on its biological activities against the insect pests is still scanty, and thus it was attempted for screening through insecticidal assay and repellent activity tests against $T$. castaneum, which is a major stored product insect pest.

\section{Materials and Methods}

Collection and preparation of test materials: Fresh plants were collected from the Rajshahi University Campus, Bangladesh followed by identification by
Prof. A.T.M. Naderuzzaman, and a voucher specimen (No. 41, 02-04-2008) was also kept in the herbarium of the Department of Botany, University of Rajshahi. After collection leaves, roots and stem were separated, and were chopped into small pieces to dry in a wellventilated room under shade. Fruits were also collected and allowed to dry. Dried materials were then powdered using a grinder, weighed and placed in separate conical flasks to add chloroform $(100 \mathrm{~g} \times$ $300 \mathrm{ml} \times 3$ times) for 48 hours. Filtrations were done by Whatman filter paper at $24 \mathrm{~h}$ interval in the same flask followed by evaporation until the extracts were left. Those were then removed to glass vials and preserved in a refrigerator at $4{ }^{\circ} \mathrm{C}$ with proper labeling.

Culture of the test insect: Five days old adult beetles of Tribolium castaneum used in the present experiment were reared in glass beakers $(500 \mathrm{ml})$ in a standard mixture of whole-wheat flour with powdered dry yeast (19:1) in an incubator at $30^{\circ} \mathrm{C} \pm 0.5^{\circ} \mathrm{C}$ without light and humidity control.

Dose-mortality test against T. castaneum: For dosemortality responses in the experiments of surface film method the doses for the $\mathrm{CHCl}_{3}$ extracts of fruits, leaves, roots and stem were confirmed through ad hoc experiments by putting 50mg of each of the extracts diluted separately in $1 \mathrm{ml}$ of solvent to apply in $50 \mathrm{~mm}$ Petri dishes and by increasing or decreasing the amount of 
extracts in repeated manners until a suitable mortality range was obtained. The doses selected for the fruit extract were $0.509,0.254,0.127,0.063,0.0318$ and $0.0159 \mathrm{mg} \mathrm{cm}^{-2}$, for the leaf extract $2.547,1.911,1.273$, $0.955,0.636$ and $0.477 \mathrm{mg} \mathrm{cm}^{-2}$, for root extract 7.643 , $5.095,2.547,1.273$ and $0.636 \mathrm{mg} \mathrm{cm}^{-2}$ and for the stem extract 4.076, 2.038, 1.019, 0.509, 0.254 and $0.127 \mathrm{mg} \mathrm{cm}^{-}$ ${ }^{2}$. Each of the five doses were diluted in $1 \mathrm{ml}$ of solvent, poured into a Petri dish and allowed to dry. Ten adult beetles were released in each of the Petri dishes, and the experiments of all the doses for each of the extracts were set in 3 replicates. The mortality of the beetles was assessed at 30min., 12, 24, 36 and 48h of exposure.

Statistical analysis of the dose-mortality data: The mortality of the beetles was recorded, while an instant observation was made just after $30 \mathrm{~min}$. of application for the detection of acute toxicity, if any. The mortality (\%) was corrected by Abbott's formula (1925). The statistical analyses were done according to Finney (1947) and Busvine (1971) to calculate the $\mathrm{LD}_{50}$ values.

Repellent activity against T. castaneum adults: The methodology for repellency test used in this experiment was adopted from the method (No. 3) of McDonald et al. (1970) with some modifications by Talukder and Howse (1993, 1994). Half filter paper discs (Whatman No. 40, diameter $9 \mathrm{~cm}$ ) were treated with the selected doses of $0.079,0.039,0.020,0.010$ and $0.005 \mathrm{mg} \mathrm{cm}^{-2}$ of fruit extract and were then attached lengthwise, edge-to-edge, to a control half-disc with adhesive tape and placed in the Petri dishes. The orientation was changed in the two remaining replicates to avoid the effects of any external directional stimulus affecting the distribution of the test insects. Ten adult insects were released in the middle of each of the filter paper circles. The same was then done for the leaf, root and stem extracts.

Observation and analysis of repellency data: Each concentration was tested for five times. Insects that settled on each of the non-treated half of the filter paper discs were counted after $1 \mathrm{~h}$ and then observed repeatedly at hourly intervals for five hours. The average of the counts was converted to percent repellency $(P R)$ using the formula of Talukder and Howse (1993, 1995): $P R=$ $(\mathrm{Nc}-5) \times 20$, where, $\mathrm{Nc}$ is the percentage of insects on the untreated half of the disc.

\section{Results and Discussion}

Dose mortality effects: The results of the dose-mortality assay revealed the fruit extract the highest in activity against the test beetles, presented in Table 1. However, the root extract, weakest among the four extracts didn't offer any mortality for $30 \mathrm{~min}$. and $12 \mathrm{~h}$ of exposure; but the $\mathrm{LD}_{50}$ values for 24,36 and $48 \mathrm{~h}$ of exposure were $6.667,5.086$ and $3.357 \mathrm{mg} \mathrm{cm}^{-2}$ respectively; and the dose-mortality activity can be arranged in the order of fruit $>$ leaf $>$ stem $>$ root extracts.

Table $1 . \mathrm{LD}_{50}$ values of fruit, leaf, root and stem extracts $\left(\mathrm{CHCl}_{3}\right)$ of $S$. ferox against $T$. castaneum adults.

\begin{tabular}{cccccc}
\hline \multirow{2}{*}{ Plant parts } & \multicolumn{5}{c}{ Duration of exposure } \\
\cline { 2 - 6 } & $30 \mathrm{~min}$. & $12 \mathrm{~h}$ & $24 \mathrm{~h}$ & $36 \mathrm{~h}$ & $48 \mathrm{~h}$ \\
\hline Fruit & 0.440 & 0.247 & 0.211 & 0.207 & 0.164 \\
Leaf & 2.394 & 1.712 & 1.540 & 1.239 & 0.993 \\
Root & - & - & 6.667 & 5.086 & 3.357 \\
Stem & 4.918 & 0.871 & 0.546 & 0.472 & 0.522 \\
\hline
\end{tabular}

Repellent effects: The repellency results are presented in Tables 2 and 3. The $\mathrm{CHCl}_{3}$ extracts of the fruit, leaf and stem of $S$. ferox offered a promising repellent effect against $T$. castaneum adults $(\mathrm{P}<0.01, \mathrm{P}<0.05$ and $\mathrm{P}<0.01$ respectively) while the root extracts did not show any repellency.

These findings receive supports from the previous researchers who carried out different biological assays including insecticidal activity tests. An alcoholic extract of the root of $S$. ferox was found active against Staphylococcus aureus and Escherichia coli (Khare, 2007). The fume produced on burning of its seed was plant inhaled to relief from toothache (Pal Singh, 2007). In Sri Lanka root of this was used as remedy in cutaneous diseases (Ahmed et al. 2009). In Philippines, leaves of the test plant are used as cataplasma for indolent swellings (Pal Singh, 2007). Because of the medicinal activity this plant has been enlisted in the ethnobotanical inventory of medicinal plants (Barbosa
Filho et al. 1991). It also has nearly the same history like other species of the same genera in context of online information. Cheryl Lans (2007) reported that these plants are used for Man's waist pain and also used for reproductive purposes. No report was found on its repellent activity among the previous findings. Being enlisted among weeds no cultivation of this plant is available even though it is a very good and promising medicinal plant and growing naturally covering a vast area of the world mostly in the tropics, and being used in the traditional and folk medicines this plant should be investigated thoroughly until furnishing the active ingredients for different health disorders. 
Table 2 Percent repulsion values and the arcsin transformed data of the fruit, leaf, root and stem extracts $\left(\mathrm{CHCl}_{3}\right)$ of $S$. ferox against $T$. castaneum adults.

\begin{tabular}{|c|c|c|c|c|c|c|}
\hline \multirow{3}{*}{ Plant part } & \multirow{3}{*}{$\underset{\mathrm{cm}^{-2}}{\operatorname{Dose}} \mathrm{mg}$} & \multicolumn{5}{|c|}{ Observation at regular intervals } \\
\hline & & $1 \mathrm{~h}$ & $2 \mathrm{~h}$ & $3 \mathrm{~h}$ & $4 \mathrm{~h}$ & $5 \mathrm{~h}$ \\
\hline & & \multicolumn{5}{|c|}{$\begin{array}{c}\text { Percent repulsion } \mathrm{PR}=(\mathrm{Nc}-5) \times 20 \\
(\text { Arcsin transformed values for ANOVA) }\end{array}$} \\
\hline \multirow{5}{*}{ Fruit } & 10 & $\begin{array}{c}60 \\
(50.77)\end{array}$ & $\begin{array}{c}100 \\
(84.84)\end{array}$ & $\begin{array}{c}93.32 \\
(75.00)\end{array}$ & $\begin{array}{c}93.32 \\
(75.00)\end{array}$ & $\begin{array}{c}100 \\
(84.84)\end{array}$ \\
\hline & 5 & $\begin{array}{l}26.66 \\
(31.05)\end{array}$ & $\begin{array}{l}26.66 \\
(31.05)\end{array}$ & $\begin{array}{c}33.32 \\
(35.24)\end{array}$ & $\begin{array}{c}26.66 \\
(31.05)\end{array}$ & $\begin{array}{c}33.32 \\
(35.24)\end{array}$ \\
\hline & 2.5 & $\begin{array}{c}0 \\
(0.00)\end{array}$ & $\begin{array}{c}33.32 \\
(35.24)\end{array}$ & $\begin{array}{c}0 \\
(0.00)\end{array}$ & $\begin{array}{c}0 \\
(0.00)\end{array}$ & $\begin{array}{c}6.66 \\
(14.89)\end{array}$ \\
\hline & 1.25 & $\begin{array}{l}26.66 \\
(31.05)\end{array}$ & $\begin{array}{c}80 \\
(63.43)\end{array}$ & $\begin{array}{c}86.66 \\
(68.53)\end{array}$ & $\begin{array}{c}6.66 \\
(14.70)\end{array}$ & $\begin{array}{c}60 \\
(50.77)\end{array}$ \\
\hline & 0.625 & $\begin{array}{c}-20 \\
(26.57)\end{array}$ & $\begin{array}{c}80 \\
(63.43) \\
\end{array}$ & $\begin{array}{l}26.66 \\
(31.05)\end{array}$ & $\begin{array}{r}86.66 \\
(68.53)\end{array}$ & $\begin{array}{c}86.66 \\
(68.53) \\
\end{array}$ \\
\hline \multirow{5}{*}{ Leaf } & 10 & $\begin{array}{l}-66.66 \\
(54.70)\end{array}$ & $\begin{array}{l}-73.34 \\
(58.89)\end{array}$ & $\begin{array}{l}-66.66 \\
(54.70)\end{array}$ & $\begin{array}{l}-86.66 \\
(68.53)\end{array}$ & $\begin{array}{c}-80 \\
(63.43)\end{array}$ \\
\hline & 5 & $\begin{array}{c}-6.66 \\
(14.89)\end{array}$ & $\begin{array}{l}-26.66 \\
(31.05)\end{array}$ & $\begin{array}{c}-20 \\
(26.57)\end{array}$ & $\begin{array}{l}-13.34 \\
(21.39)\end{array}$ & $\begin{array}{c}13.34 \\
(21.39)\end{array}$ \\
\hline & 2.5 & $\begin{array}{c}0 \\
(0.00)\end{array}$ & $\begin{array}{c}0 \\
(0.00)\end{array}$ & $\begin{array}{c}-20 \\
(26.57)\end{array}$ & $\begin{array}{c}-46.66 \\
(43.05)\end{array}$ & $\begin{array}{c}-40 \\
(39.23)\end{array}$ \\
\hline & 1.25 & $\begin{array}{c}-40 \\
(39.23)\end{array}$ & $\begin{array}{c}6.66 \\
(14.89)\end{array}$ & $\begin{array}{l}-26.66 \\
(31.05)\end{array}$ & $\begin{array}{c}-26.66 \\
(31.05)\end{array}$ & $\begin{array}{c}-20 \\
(26.57)\end{array}$ \\
\hline & 0.625 & $\begin{array}{c}-20 \\
(26.57)\end{array}$ & $\begin{array}{c}-26.66 \\
(31.05)\end{array}$ & $\begin{array}{c}-66.66 \\
(54.70)\end{array}$ & $\begin{array}{c}-53.34 \\
(46.89)\end{array}$ & $\begin{array}{l}-33.34 \\
(35.24)\end{array}$ \\
\hline \multirow{5}{*}{ Root } & 10 & $\begin{array}{c}-40 \\
(39.23)\end{array}$ & $\begin{array}{l}-26.68 \\
(31.05)\end{array}$ & $\begin{array}{c}-33.34 \\
(35.24)\end{array}$ & $\begin{array}{c}-33.34 \\
(35.24)\end{array}$ & $\begin{array}{l}-33.34 \\
(35.24)\end{array}$ \\
\hline & 5 & $\begin{array}{c}-40 \\
(39.23)\end{array}$ & $\begin{array}{l}-26.68 \\
(31.05)\end{array}$ & $\begin{array}{c}-20 \\
(26.57)\end{array}$ & $\begin{array}{c}-13.34 \\
(21.39)\end{array}$ & $\begin{array}{c}-20 \\
(26.57)\end{array}$ \\
\hline & 2.5 & $\begin{array}{l}-13.34 \\
(21.39)\end{array}$ & $\begin{array}{c}0 \\
(0.00)\end{array}$ & $\begin{array}{c}-40 \\
(39.22)\end{array}$ & $\begin{array}{c}33.34 \\
(35.24)\end{array}$ & $\begin{array}{c}33.34 \\
(35.24)\end{array}$ \\
\hline & 1.25 & $\begin{array}{c}0 \\
(0.00)\end{array}$ & $\begin{array}{c}13.32 \\
(21.39)\end{array}$ & $\begin{array}{c}-6.68 \\
(14.89)\end{array}$ & $\begin{array}{c}-6.68 \\
(14.89)\end{array}$ & $\begin{array}{c}0 \\
(0.00)\end{array}$ \\
\hline & 0.625 & $\begin{array}{c}20 \\
(26.57) \\
\end{array}$ & $\begin{array}{c}40 \\
(39.23)\end{array}$ & $\begin{array}{c}20 \\
(26.57)\end{array}$ & $\begin{array}{c}33.32 \\
(35.24)\end{array}$ & $\begin{array}{c}26.66 \\
(31.05) \\
\end{array}$ \\
\hline \multirow{5}{*}{ Stem } & 10 & $\begin{array}{c}40 \\
(39.23)\end{array}$ & $\begin{array}{c}-33.34 \\
(35.24)\end{array}$ & $\begin{array}{c}-33.34 \\
(35.24)\end{array}$ & $\begin{array}{c}-40 \\
(39.23)\end{array}$ & $\begin{array}{c}-40 \\
(39.23)\end{array}$ \\
\hline & 5 & $\begin{array}{c}66.66 \\
(54.70)\end{array}$ & $\begin{array}{c}-53.34 \\
(46.89)\end{array}$ & $\begin{array}{l}-86.66 \\
(68.53)\end{array}$ & $\begin{array}{c}-100 \\
(84.84)\end{array}$ & $\begin{array}{l}-86.66 \\
(68.53)\end{array}$ \\
\hline & 2.5 & $\begin{array}{c}40 \\
(39.23)\end{array}$ & $\begin{array}{c}-33.34 \\
(35.24)\end{array}$ & $\begin{array}{c}-53.34 \\
(46.89)\end{array}$ & $\begin{array}{l}-86.66 \\
(68.53)\end{array}$ & $\begin{array}{c}-80 \\
(63.43)\end{array}$ \\
\hline & 1.25 & $\begin{array}{c}6.66 \\
(14.89)\end{array}$ & $\begin{array}{c}-26.66 \\
(31.05)\end{array}$ & $\begin{array}{c}-20 \\
(26.57)\end{array}$ & $\begin{array}{c}-26.66 \\
(31.05)\end{array}$ & $\begin{array}{c}-33.34 \\
(35.24)\end{array}$ \\
\hline & 0.625 & $\begin{array}{c}-6.66 \\
(14.89)\end{array}$ & $\begin{array}{c}6.66 \\
(14.89)\end{array}$ & $\begin{array}{c}6.66 \\
(14.89)\end{array}$ & $\begin{array}{c}-6.66 \\
(14.89)\end{array}$ & $\begin{array}{c}6.66 \\
(14.89)\end{array}$ \\
\hline
\end{tabular}


Table 3. Repellency effect of fruit, leaf, root and stem extracts $\left(\mathrm{CHCl}_{3}\right)$ of $S$. ferox against $T$. castaneum adults.

\begin{tabular}{ccc}
\hline \multirow{2}{*}{ Extract } & Between time interval $(\mathrm{df}=4)$ & Between doses $(\mathrm{df}=4)$ \\
\cline { 2 - 3 } & F-value & F-value \\
\hline Fruit & 4.226 & $22.065^{\star \star}$ \\
Leaf & 2.014 & $10.457^{\star}$ \\
Root & 0.162 & 4.435 \\
Stem & 2.699 & $22.1993^{\star \star}$ \\
\hline
\end{tabular}

$*=$ significant at $5 \%$ level; $* *=$ significant at $1 \%$ level

Acknowledgements: The authors are thankful to the University of Rajshahi for a UGC supported research grant and also to the Chairman, Department of Zoology, University of Rajshahi, Bangladesh for providing laboratory facilities.

\section{References}

Abbott, WS. 1925. A method of computing the effectiveness of an insecticide. $J$ Econ Entomol 18,265-267.

Ahmed, ZU, Hassan, MA Begum, ZNT Khondker, M Kabir, SMH, Ahmad, M, Ahmed ATA. 2009. Encyclopedia of Flora and Fauna of Bangladesh. Asiatic Society of Bangladesh. 10,309-310.

Barbosa Filho, JM, Agra, MF, Oliseeira, RA, Paulo, MQ, Trolin, G, Cunha, EV, Ataide, JR, Bhattacharyya, J. 1991. Chemical and pharmacological investigation of Solanum species of Brazil- a search for solasodine and other potentially useful therapeutic agents. Mem-Ins-Oswaldo-Cruz 86,2189-2191.

Busvine, JR. 1971. A critical review of the techniques for testing insecticides. Commonwealth Agricultural Buereux, London. $345 \mathrm{pp}$.

Cheryl Lans. 2007. Ethnomedicines used in Trinidad and Tobago for reproductive problems. J Ethnobiol Ethnomed 3,13.

Finney, DJ. 1947. Probit analysis: a statistical treatment of the sigmoid response curve. Cambridge University Press, London. 333 p.
Joy, PP, Thomas, J, Mathew, S, Skaria, BP. 2001. Medicinal Plants. Tropical Horticulture Vol. 2. (eds. Bose, TK, Kabir, J, Das, P, Joy, PP). Naya Prokash, Calcutta, pp.449-632.

Khare, CP. 2007. Indian Medicinal Plants: An Illustrated Dictionary. Springer Verlag, Heidelberg. $836 \mathrm{p}$.

Kirtikar, KR, Basu, BD. 1935. Indian Medicinal plants, Vol. III, 2nd. ed. Basu, LM, Allahabad, India. pp.1593-2393.

McDonald, LL, Guy, RH, Speirs, RD. 1970. Preliminary evaluation of new candidate materials as toxicants, repellents and attractants against stored-product insects. Marketing Research Report No. 882. Agricultural Research Service, US Department of Agriculture, Washington, DC

Pal Singh, A. 2007. Lakśmanā-Āyurvedic Drug of Controversial origin. Ethnobot Leaflets 11,141-147.

Talukder, FA, Howse, PE. 1993. Deterrent and insecticidal effects of extracts of pithraj, Aphanamixis polystachya (Meliaceae) against Tribolium castaneum in storage. J Chem Ecol 19,2463-2471.

Talukder, FA, Howse, PE. 1994. Repellent, toxic and food protectant effect of Pithraj, Aphanamixis polystachya extracts against pulse beetle, Callosobruchus chinensis in storage. J Chem Ecol 20,899-908.

Talukder, FA, Howse, PE. 1995. Evaluation of Aphanamixis polystachya as a source of repellents, antifeedants, toxicants and protectants in storage against Tribolium castaneum (Herbs). J Stored Prod Res $31,55-61$.

Manuscript received on 8 July 2012 and revised on 17 November 2012 